\title{
EXPLORING CLOUD-BASED PLATFORMS FOR RAPID INSAR TIME SERIES ANALYSIS
}

\author{
A. Piter ${ }^{1, *}$, M. Vassileva ${ }^{2,1}$, M. Haghshenas Haghighi ${ }^{1}$, M. Motagh ${ }^{1,2}$ \\ ${ }^{1}$ Institute of Photogrammetry and GeoInformation, Leibniz Universität Hannover, Germany \\ (piter,mahmud)@ipi.uni-hannover.de \\ ${ }^{2}$ GFZ German Research Centre for Geosciences, Section of Remote Sensing and Geoinformatics, 14473 Potsdam, Germany \\ (magda88, motagh)@gfz-potsdam.de
}

KEY WORDS: Cloud platform, InSAR, Big data, Time series analysis, Deformation

\begin{abstract}
:
The idea of near real-time deformation analysis using Synthetic Aperture Radar (SAR) data as a response to natural and anthropogenic disasters has been an interesting topic in the last years. A major limiting factor for this purpose has been the non-availability of both spatially and temporally homogeneous SAR datasets. This has now been resolved thanks to the SAR data provided by the Sentinel-1A/B missions, freely available at a global scale via the Copernicus program of the European Space Agency (ESA). Efficient InSAR analysis in the era of Sentinel demands working with cloud-based platforms to tackle problems posed by large volumes of data. In this study, we explore a variety of existing cloud-based platforms for Multi-Temporal Interferometric SAR (MTI) analysis and discuss their opportunities and limitations.
\end{abstract}

\section{INTRODUCTION}

By the year 2021, more than 6 million Sentinel-1 C-band SAR products are available, representing $10 \mathrm{~PB}$ of data. In 2022 NASA-ISRO SAR (NISAR) mission will be launched, providing 3 to $10-\mathrm{m}$ resolution $\mathrm{L}$ and $\mathrm{S}$-band image acquisitions with a revisit time of 12 days. This mission will further increase the volume of SAR data that are freely available and open to the community to be used in hazard and climate change-related applications. Currently, well-established MTI methods such as Persistent Scatterer (PS) (Ferretti et al., 2001) and Small BAseline Subset (SBAS) (Berardino et al., 2002) allow multitemporal displacement analysis to detect temporal and spatial changes of surface deformation using SAR data.

The interest in near real-time (Spaans and Hooper, 2016, Ansari et al., 2017) and nation-wide deformation analysis (Haghshenas Haghighi and Motagh, 2017, Kalia et al., 2017, De Luca et al., 2017) poses a computational challenge for traditional processing using local desktops and clusters. The need for large data storage and computational power are the main challenges the scientific community is facing nowadays. Apart from providing ourselves with local high-performing computer infrastructures and huge storage hard drivers, solutions that require significant economical resources and good IT maintenance skills, the most efficient perspective would be exploiting cloud-processing services. The latter refers to the on-demand availability of flexible computing power and data storage over the "Internet", which are physically hosted in data centers and server farms around the world.

In the era of exponentially increasing Earth Observation (EO) data volume (big data), cloud-based data management solutions guarantee direct access to the data, without the need of downloading and storing them on local servers, which is one of the main advantages of using these services. In the specific case of EO applications, a particular need is to have on

\footnotetext{
* Corresponding author
}

the same cloud environment a direct access to the satellite data archives, the processing tools and the computing power at the same time. Nowadays, several cloud-platform services, connected to the freely available EO data archives and designed for global, European, or single-national scale analysis are available to different users. However, their full potential and effective operativity have not been explored by the scientific community yet. This may be on the one hand due to the still experimental nature of such EO data designed services and on the other hand due to the necessity still to establish a long-term economic sustainability program and clear user policy.

In this work, we exploited and discussed the capabilities of three cloud-computing platforms that can be used to perform MTI analysis: the ESA's Geohazards Exploitation Platform (GEP), CODE-DE, and the ASF's OpenSARLab. Moreover, we investigate the automatically processed and publicly available products of the COMET-LiCSAR project. In the following, we provide a comparison between their different specifications and present some relevant case studies for suitable applications.

\section{CLOUD-BASED PLATFORMS FOR MTI ANALYSIS}

We analysed the performances and the specifications of the aforementioned platforms and products, with the primary goal to highlight strengths and weaknesses and identify the most suitable application for each of them. To do so, we considered and discussed the following aspects:

- access for users: administration, science, private

- access to the data: real time, historic, area, auxiliary data

- processing performances: software availability, time of processing, changing parameters, quality of results

\subsection{GEP}

$\mathrm{GEP}^{1}$ started as one of the ESA's Thematic Exploitation Platforms initiative, and is developed under a consortium led by

\footnotetext{
1 https://geohazards-tep.eu
} 
Terradue, specifically aimed to support the exploitation of satellite data for geohazards. The platform provides a rich set of ready to use EO Data processing services for geohazards analysis and monitoring (Foumelis et al., 2021). One of the services in GEP is P-SBAS, Parallel Small BAseline Subset, developed by CNR IREA for time-series analysis of ground deformation (De Luca et al., 2015, Manunta et al., 2019). It is a SBAS processing chain that runs in parallel computing. All the GEP processing services rely on several Copernicus Data and Information Access Services (DIAS) and Information and communications technology (ICT) providers. Through GEP there is a direct connection to the Copernicus SciHub server, which contains the online Sentinel-1 data archive. Due to the large amount of data, only acquisitions from the last year are kept online, while older data are stored in an offline Long Term Archive. These data can be requested at any time, though it is a process that requires some time (normally a few hours). After a long period where free access was granted to a small community of early adopters inside the scientific community, today the GEP services are available on a pay-per-use basis to the public. In addition, the ESA Network of Resources (NoR) initiative provides sponsorship for the purchases of the GEP services access.

\subsection{CODE-DE}

The cloud computing platform CODE-DE ${ }^{2}$ is provided by the Federal Ministry of Transport and Digital Infrastructure (BMVI) and the German Aerospace Center (DLR). The platform is designed as "infrastructure as a service" (Figure 1). CODE-DE offers virtual machines for scalable applications and a JupyterHub for interactive use of the platform. Here, users can install software, develop process chains and upload their code to the platform. The high customizability is a strength of this cloud computing environment, which makes the platform useful for various applications over the spatial extent of Germany.

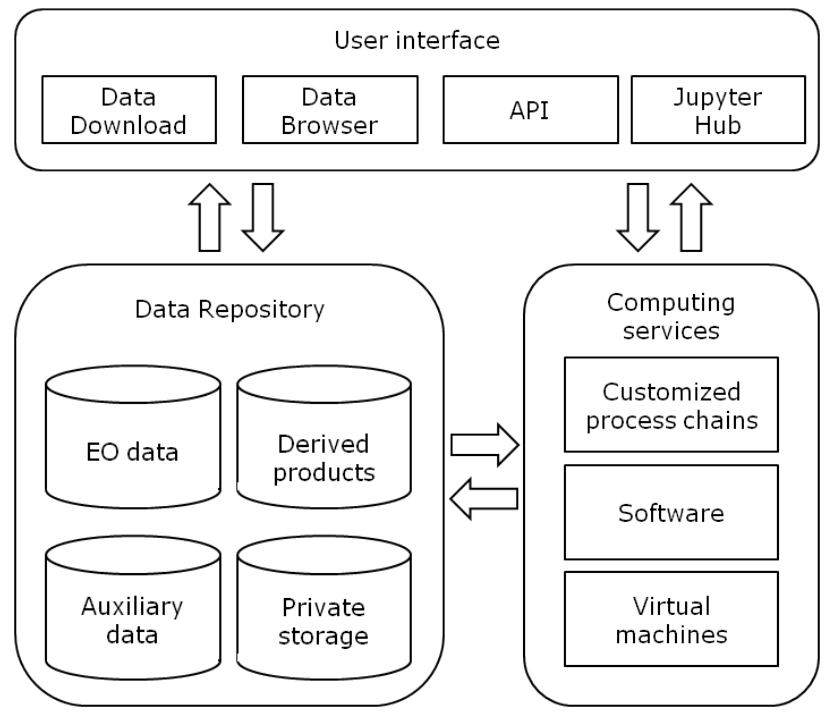

Figure 1. General scheme of a cloud service for InSAR processing. The presented cloud services vary mostly in the specification of the user interface and the availability of the computing services.

The platform CODE-DE is designed for German federal and state authorities to access and process the Copernicus data.

2 https://code-de.org/en/
However, the platform is free to use for everyone, but is prioritized based on four categories that favour authorities over private users. An application is required for access and configuration of computational resources on a virtual machine. Moreover, JupyterHub is available for every registered user without need for applying for a contingent of resources.

CODE-DE provides an own archive with a broad variety of EO data from different sensors. Among these, the data from the radar mission Sentinel-1 are available. Moreover, the common auxiliary data needed for InSAR processing such as the DEM and precise orbit files are provided. The archive covers both the recent acquisitions of Sentinel-1 in near real-time and the historic data since the start of the mission. However, the historical data is limited to the spatial extent of Germany, while the data from the last six months are available for the rest of the world.

The platform CODE-DE offers its users high customizability. In general, no InSAR software is already preinstalled there, but it can be installed on a virtual machine by those users having access to a contingent of resources. Process chains can be deployed and jupyter notebooks enable further development on the platform. InSAR processing on CODE-DE is neither limited to specific software nor predefined parameters and thus, CODE-DE is particularly valuable for addressing scientific questions. This implies also that the quality of the results is not governed by the cloud computing platform CODE-DE but the software used. The processing time on the platform can be reduced by exploiting parallel computation on multiple cores.

\subsection{OpenSARLab}

OpenSARLab $^{3}$, operated by the National Aeronautics and Space Administration's (NASA) Alaska Satellite Facility (ASF), is a jupyter notebook-based SAR Data Analysis Platform hosted in the Amazon Web Services (AWS) Cloud. It has direct access to the entire ASF data archive. Apart from Sentinel-1 data, the archive also includes several pastoperational SAR missions such as ALOS-1, Radarsat-1, and ERS. In the specific case of Sentinel-1 data, the main advantage is that the entire Sentinel-1 archive is instantly available. However, since the ASF Distributed Active Archive Center is waiting to get the product from ESA, it can occur that for newly released Sentinel-1 scenes, their availability on ASF is delayed compared to the Copernicus SciHub server.

A very important advantage for prompt MTI analysis is the NASA Jet Propulsion Laboratory (JPL) Advanced Rapid Imaging and Analysis (ARIA) system, which provides $90 \mathrm{~m}$ spatial resolution Standard Displacement products (Geocoded Unwrapped Interferograms, GUW), derived from Sentinel-1 data for certain locations starting from the beginning of the data archive (Bekaert et al., 2019). The ARIA products are also freely available in the ASF DAAC archive. Unfortunately, these products are only a prototype for the upcoming NISAR mission and not part of a long-term plan, and their production is not guaranteed. However, having those products for certain areas of active tectonics and volcanoes and regions of large scale geomorphological processes such as Philippines, Galapagos, Hawaii, California etc, enables us to timely describe the historical situation of deformation over large areas.

The open-source software ISCE (Rosen et al., 2012, Fattahi et al., 2017), MintPy (Yunjun et al., 2019) and ARIAtools (Buzzanga et al., 2020) are pre-installed and ready to be

\footnotetext{
3 https://opensarlab.asf .alaska.edu
} 
used inside OpenSARLab. The platform is currently only available to a small group of beta testers that have been authorized by NASA, with a limited computing power availability, though a near future goal is to open up its access to a broader audience.

\subsection{LiCSAR}

Centre for the Observation and Modelling of Earthquakes, Volcanoes and Tectonics (COMET) ${ }^{4}$ provides automatically generated interferograms and coherence maps of Sentinel-1 mainly in tectonic and volcanic areas around the world. The geocoded LiCSAR products have a spatial resolution of approximately 100 meters and are freely available for download. Within two weeks of new SAR acquisition, new products are generated using supercomputing facilities and available on the platform for download (Lazeckỳ et al., 2020).

LiCSBAS is an open-source package available to perform InSAR time series analysis using LiCSAR products (Morishita et al., 2020). The package performs different steps, from downloading the data, to quality check, time series analysis, and postprocessing of the results. It can be installed on local computers or cloud platforms, but in either case it still requires downloading the interferometric products from the LiCSAR platform.

\section{CASE STUDIES}

The potential of cloud-based MTI processing is demonstrated by several case studies conducted on the different cloud platforms. Since for each platform the processing chains vary a lot in term of customizability and also the data access is different in terms of spatial and temporal coverage, we decided to design a specific case studies for each of them in order to highlight their specific advantages.

\subsection{Geohazard monitoring for large areas (GEP)}

As already mentioned, the cloud-processing services are physically hosted in data centers around the world. Specifically GEP platform is hosted in the OVHcloud infrastructure in Strasbourg. This infrastructure suffered from a major fire incident on March 10, 2021, which interrupted access to the data and Virtual Machines supporting the P-SBAS processing on-demand service. Therefore, at the time of writing this paper we were not able to run our own P-SBAS analysis on GEP. To the best of our knowledge, the Sentinel-1 data access has already been completely restored, though the P-SBAS tool is still out of access. A few studies have already shown the capability of this system for efficient MTI analysis using a variety of SAR sensors (Galve et al., 2017, Cigna and Tapete, 2021).

\subsection{Mining monitoring (CODE-DE)}

On CODE-DE we exemplarily investigate mining induced deformations in the Rhenish coal fields in midwest Germany (Tang et al., 2020). For that purpose, we installed two open source InSAR software packages on a virtual machine on CODE-DE. First, a stack of unwrapped differential interferograms was created using ISCE (Rosen et al., 2012, Fattahi et al., 2017) and second, the MTI analysis was performed using the SBAS method implemented in the software MintPy (Yunjun et al., 2019).

\footnotetext{
4 https://comet.nerc.ac.uk/comet-lics-portal/
}

For our case study a set of 53 Sentinel-1 images from ascending orbit were processed covering one year beginning from September 2019. The whole processing was performed in less than a day. Clear deformation signals are visible at the open pit mine (Figure 2) with up to $10 \mathrm{~cm} /$ year and $4 \mathrm{~cm} /$ year subsidence and uplift rates in line-of-sight (LOS), respectively. The deformation map also shows subsidence in regions nearby affecting traffic infrastructure like highways and railway tracks. Based on these results, further scientific investigations can reveal detailed information on the impact of mining on the traffic infrastructure. On CODE-DE, past deformations can be analysed as well as recent acquisitions can be integrated into a near-real time monitoring system running on the cloud-computing environment.

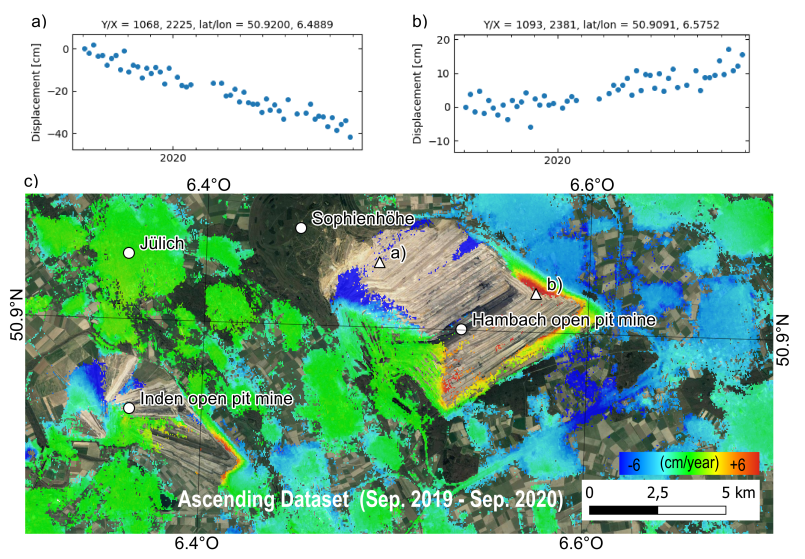

Figure 2. Mining induced deformation at the Rhenish coal fields (Germany) derived from Sentinel-1 ascending track images for the period September 2019 to September 2020. a) and b) depict exemplary LOS displacement time series in c). c) shows the average displacement velocity map along the LOS direction; white triangles indicate the locations of the plotted time series in

a) and b). A coherence mask greater than 0.7 was applied.

Background imagery for c) Google Earth GeoBasis-DE/BKG, Map data (C2021.

\subsection{MTI analysis using ARIA products (OpenSARLab)}

We used the available ARIA products to assess ground subsidence around Manila Bay (Luzon island, Philippines) for the period October 2014 - March 2020. This deltaic and coastal environment is well known for being subjected to subsidence due to both autocompaction and groundwater exploitation (Raucoules et al., 2013). Unwrapped products derived from both ascending and descending Sentinel-1 acquisitions were available for the period between October 2014 and March 2020: for ascending path 142 a total of 324 interferograms (S1GUNW-A-R-142, ASF DAAC 2021, contains modified Copernicus Sentinel data 2021, processed by ESA) and for descending path 32 a total of 448 interferograms (S1-GUNW-A-R-032, ASF DAAC 2021, contains modified Copernicus Sentinel data 2021 , processed by ESA) were available and used in the timeseries processing. The average displacement velocity maps along the LOS direction in both geometries is clearly showing large subsiding regions with a rate up to $10 \mathrm{~cm} /$ year (Figure 3). By using ARIA products and MintPy in OpenSARLab, we were able to detect in just a few hours the historical displacement conditions over a large region, providing a very good overview of the possible geohazard affecting that specific area. Although an up to date analysis is not possible using ARIA products, 
it can always be integrated by processing separately the final missing Sentinel-1 time-series.
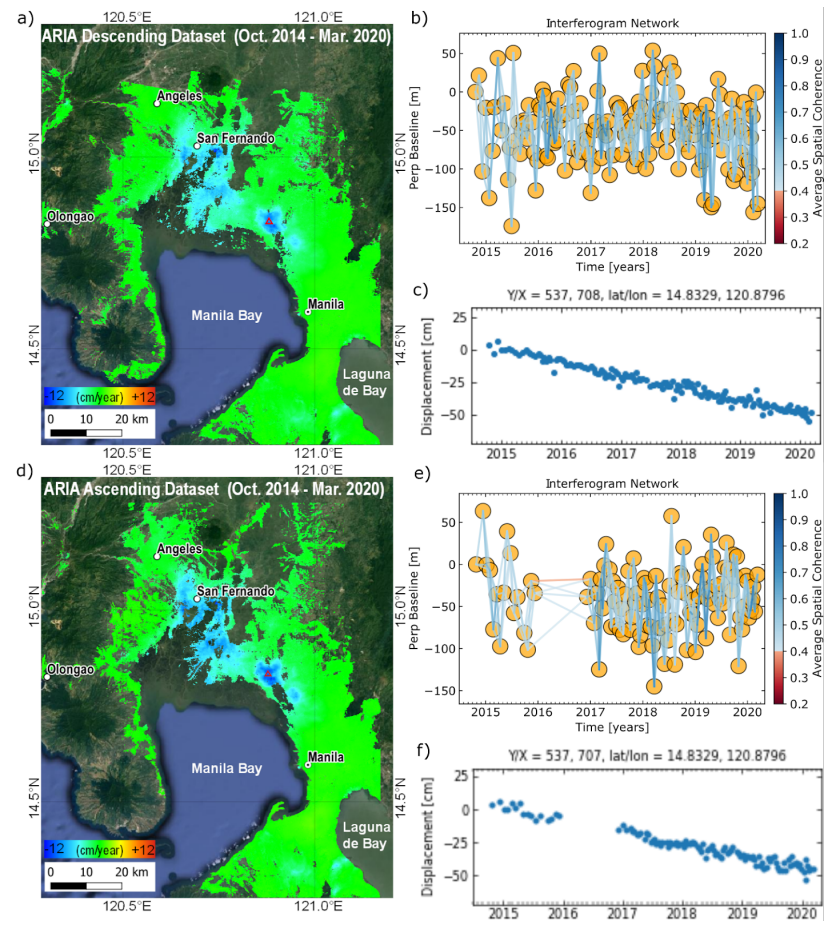

Figure 3. MintPy processed displacement time series over

Manila region (Philippines) derived from Sentinel-1 ARIA products for the period between October 2014 and March 2020. a) and d) average displacement velocity maps along the LOS direction for descending and ascending orbit path acquisitions. A coherence mask greater than 0.6 was applied. Red triangles represent the displacement time series plotted in c) and f). b) and

e) network of interferograms for descending and ascending datasets. Background imagery for a) and b) Google Earth Image Landsat/Copernicus, Map data @2021.

\subsection{MTI analysis using COMET-LiCSAR products}

We used the available LiCSAR Sentinel-1 InSAR products to assess surface deformation in Tehran, the capital of Iran, which has been subject to long-term subsidence caused by groundwater extraction (Motagh et al., 2008, Haghighi and Motagh, 2019). LiCSAR regularly generates unwrapped interferograms and coherence maps for this area in both ascending and descending tracks of Sentinel-1. We use more than 400 interferograms in a descending path from six years of Sentinel-1 data and analyzed them using SBAS time series analysis in LiCSBAS software on a local computer. The average subsidence rate map indicates a large area to the southwest of Tehran is subject to a land subsidence with a rate exceeding $15 \mathrm{~cm} /$ year in satellite line of sight (Figure 4). The results are in general consistent with other research in this area and confirms that the subsidence is still ongoing without significant change in the longterm trend. It is worth noting that, due to $100-\mathrm{m}$ resolution of original interferograms, maximum surface deformation might be underestimated compared to the cases, where full-resolution interferograms are used for subsidence analysis, for example using PS or SBAS at single look resolution (Hooper et al., 2007, Hooper, 2008).

Using the LiCSAR products, it is not only possible to achieve a quick overview of the historical situation of deformation in the past six years, but it is also possible to update the results within two weeks after new SAR data is acquired. An advantage of LiCSAR products is that one of the most time-consuming steps of InSAR time series pipeline, which is the production and unwrapping of the interferograms, is already performed on supercomputing facilities. The actual time series analysis step can then be performed in a few hours. Therefore, LiCSBAS is suitable for applications like large-scale deformation analysis (Morishita et al., 2020, Weiss et al., 2020).

Moreover, it is possible to run LiCSBAS on cloud platforms to increase the computational power and obtain fast results over large areas. However, downloading the interferograms from the LiCSAR platform is still a bottleneck and will be dependent on LiCSAR servers' availability and bandwidth. Another limitation of LiCSAR products is that they have a fixed multilooking factor. Therefore, for specific analysis, e.g. localized deformation of buildings or bridges (Shamshiri et al., 2014), the signal might be averaged out in the multilooked interferograms. For large-scale deformation signals, e.g. land subsidence due to groundwater extraction or tectonic signals, LiCSAR is a great data treasure.
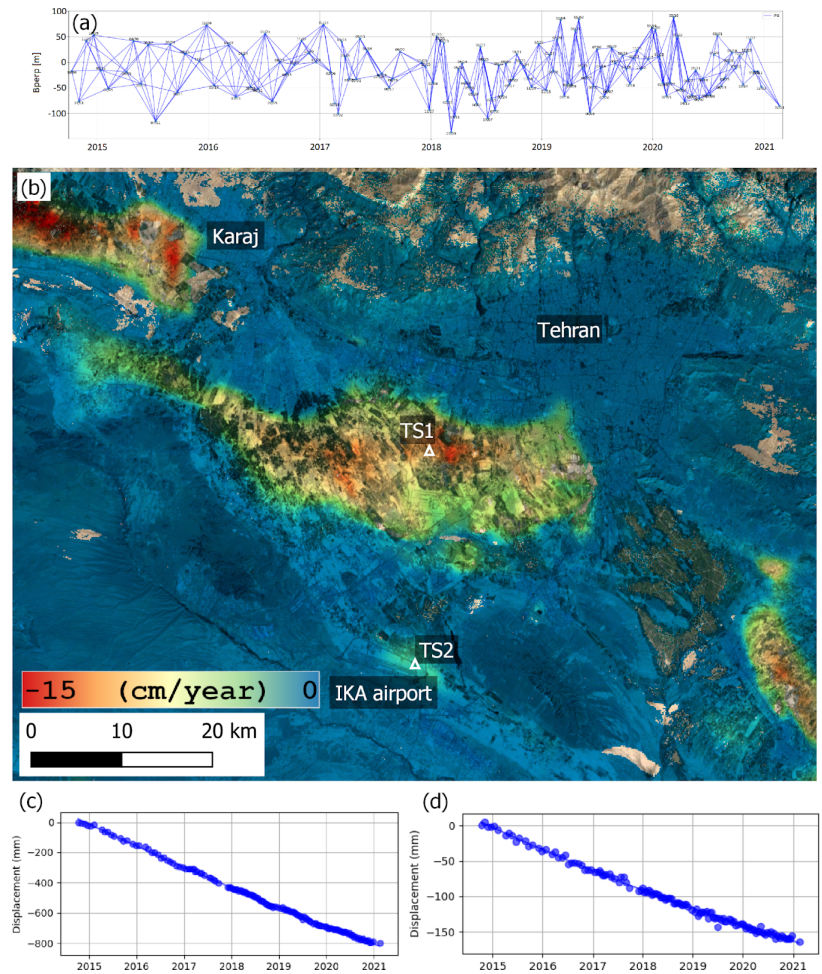

Figure 4. InSAR time series analysis of LiCSAR products in Tehran between 2014 and 2021. (a) network of interferograms. (b) linear rate of displacement in satellite LOS direction. (c) and (d) time series of displacement at sample points indicated by TS1 and TS2 in (b). The average rate of displacement indicates several subsidence areas near Tehran with a peak exceeding $15 \mathrm{~cm} /$ year. (c) and (d) show two examples of deformation time series at locations indicated by TS1 and TS2. TS1 is located in the main subsidence bowl to the southwest of Tehran. TS2 is located near Tehran's international airport. Background imagery for (b) Google Earth Image Landsat/Copernicus, Map data (C)2021. 


\section{CONCLUSION AND OUTLOOKS}

In our work we exploited three main cloud-computing platforms specifically for MTI analysis using Sentinel-1 data: the Geohazards Exploitation Platform (GEP), CODE-DE, OpenSARLab. The main advantages are the direct access to free available EO data, computational resources, and advanced InSAR processing tools. In all platforms there is direct access to Sentinel-1 data. Specifically, CODE-DE provides full data coverage over Germany; for the rest of the world the access is limited to the last six months. Both GEP and OpenSARLab have full access to Sentinel-1 all over the world with the disadvantage in the case of the GEP having immediate access to only the final year of Sentinel-1 acquisitions, and a specific request has to be sent to obtain old archived data. GEP and OpenSARLab also provide a variety of pre-installed processing tools ready to be used, while CODE-DE offers virtual machines for scalable applications and a JupyterHub for interactive use of the platform.

Moreover already processed interferogram products such as ARIA and COMET are available for some specific areas of interest. In case of ARIA products, data can be easily accessed and processed using MintPy software. COMET LiCSAR platform is an automatic processor, developed to access and process COMET data. Using such tools it is possible to rapidly perform analysis of deformation at a medium resolution (90100 meters).

Our future work includes the project SAR4Infra funded by the Federal Ministry of Transport and Digital Infrastructure and DLR. It aims at developing an automated system for assessing the stability of traffic infrastructure in Germany in near realtime. An automated InSAR time series processing chain will be implemented on CODE-DE, as it offers great customizability and data availability over Germany which is required for the research project. Notably, CODE-DE is primarily dedicated to the operational users of our process chain, the German federal and state authorities, and thus CODE-DE is a key element for the success of the project.

\section{REFERENCES}

Ansari, H., De Zan, F., Bamler, R., 2017. Sequential estimator: Toward efficient InSAR time series analysis. IEEE Transactions on Geoscience and Remote Sensing, 55(10), 5637-5652.

Bekaert, D. P., Karim, M., Linick, J. P., Hua, H., Sangha, S., Lucas, M., Malarout, N., Agram, P. S., Pan, L., Owen, S. E. et al., 2019. Development of open-access standardized InSAR displacement products by the Advanced Rapid Imaging and Analysis (ARIA) project for natural hazards. AGU Fall Meeting Abstracts, 2019, G23A-04.

Berardino, P., Fornaro, G., Lanari, R., Sansosti, E., 2002. A new algorithm for surface deformation monitoring based on small baseline differential SAR interferograms. IEEE Transactions on geoscience and remote sensing, 40(11), 2375-2383.

Buzzanga, B., Bekaert, D. P., Hamlington, B. D., Sangha, S. S., 2020. Toward Sustained Monitoring of Subsidence at the Coast Using InSAR and GPS: An Application in Hampton Roads, Virginia. Geophysical Research Letters, 47(18), e2020GL090013.
Cigna, F., Tapete, D., 2021. Sentinel-1 BigData Processing with P-SBAS InSAR in the Geohazards Exploitation Platform: An Experiment on Coastal Land Subsidence and Landslides in Italy. Remote Sensing, 13(5), 885.

De Luca, C., Cuccu, R., Elefante, S., Zinno, I., Manunta, M., Casola, V., Rivolta, G., Lanari, R., Casu, F., 2015. An ondemand web tool for the unsupervised retrieval of Earth's surface deformation from SAR data: The P-SBAS service within the ESA G-POD environment. Remote Sensing, 7(11), 1563015650 .

De Luca, C., Zinno, I., Manunta, M., Lanari, R., Casu, F., 2017. Large areas surface deformation analysis through a cloud computing P-SBAS approach for massive processing of DInSAR time series. Remote Sensing of Environment, 202, 3-17.

Fattahi, H., Agram, P., Simons, M., 2017. A network-based enhanced spectral diversity approach for TOPS time-series analysis. IEEE Transactions on Geoscience and Remote Sensing, 55(2), 777-786.

Ferretti, A., Prati, C., Rocca, F., 2001. Permanent scatterers in SAR interferometry. IEEE Transactions on geoscience and remote sensing, 39(1), 8-20.

Foumelis, M., Papazachos, C., Papadimitriou, E., Karakostas, V., Ampatzidis, D., Moschopoulos, G., Kostoglou, A., Ilieva, M., Minos-Minopoulos, D., Mouratidis, A. et al., 2021. On rapid multidisciplinary response aspects for Samos 2020 M7.0 earthquake. Acta Geophysica, 1-24.

Galve, J. P., Pérez-Peña, J. V., Azañón, J. M., Closson, D., Caló, F., Reyes-Carmona, C., Jabaloy, A., Ruano, P., Mateos, R. M., Notti, D. et al., 2017. Evaluation of the SBAS InSAR service of the European Space Agency's Geohazard Exploitation Platform (GEP). Remote Sensing, 9(12), 1291.

Haghighi, M. H., Motagh, M., 2019. Ground surface response to continuous compaction of aquifer system in Tehran, Iran: Results from a long-term multi-sensor InSAR analysis. Remote sensing of environment, 221, 534-550.

Haghshenas Haghighi, M., Motagh, M., 2017. Sentinel-1 InSAR over Germany: Large-scale interferometry, atmospheric effects, and ground deformation mapping. ZfV: Zeitschrift für Geodäsie, Geoinformation und Landmanagement, 2017(4), 245-256.

Hooper, A., 2008. A multi-temporal InSAR method incorporating both persistent scatterer and small baseline approaches. Geophysical Research Letters, 35(16).

Hooper, A., Segall, P., Zebker, H., 2007. Persistent scatterer interferometric synthetic aperture radar for crustal deformation analysis, with application to Volcán Alcedo, Galápagos. Journal of Geophysical Research: Solid Earth, 112(B7).

Kalia, A., Frei, M., Lege, T., 2017. A Copernicus downstreamservice for the nationwide monitoring of surface displacements in Germany. Remote Sensing of Environment, 202, 234-249.

Lazeckỳ, M., Spaans, K., González, P. J., Maghsoudi, Y., Morishita, Y., Albino, F., Elliott, J., Greenall, N., Hatton, E., Hooper, A. et al., 2020. LiCSAR: An automatic InSAR tool for measuring and monitoring tectonic and volcanic activity. Remote Sensing, 12(15), 2430. 
Manunta, M., De Luca, C., Zinno, I., Casu, F., Manzo, M., Bonano, M., Fusco, A., Pepe, A., Onorato, G., Berardino, P. et al., 2019. The parallel SBAS approach for Sentinel-1 interferometric wide swath deformation time-series generation: Algorithm description and products quality assessment. IEEE Transactions on Geoscience and Remote Sensing, 57(9), 62596281.

Morishita, Y., Lazecky, M., Wright, T. J., Weiss, J. R., Elliott, J. R., Hooper, A., 2020. LiCSBAS: An open-source InSAR time series analysis package integrated with the LiCSAR automated Sentinel-1 InSAR processor. Remote Sensing, 12(3), 424.

Motagh, M., Walter, T. R., Sharifi, M. A., Fielding, E., Schenk, A., Anderssohn, J., Zschau, J., 2008. Land subsidence in Iran caused by widespread water reservoir overexploitation. Geophysical Research Letters, 35(16).

Raucoules, D., Le Cozannet, G., Wöppelmann, G., De Michele, M., Gravelle, M., Daag, A., Marcos, M., 2013. High nonlinear urban ground motion in Manila (Philippines) from 1993 to 2010 observed by DInSAR: implications for sea-level measurement. Remote sensing of environment, 139, 386-397.

Rosen, P. A., Gurrola, E., Sacco, G. F., Zebker, H., 2012. The InSAR scientific computing environment. EUSAR 2012; 9th European Conference on Synthetic Aperture Radar, VDE, 730 733 .

Shamshiri, R., Motagh, M., Baes, M., Sharifi, M. A., 2014. Deformation analysis of the Lake Urmia causeway (LUC) embankments in northwest Iran: insights from multi-sensor interferometry synthetic aperture radar (InSAR) data and finite element modeling (FEM). Journal of Geodesy, 88(12), 11711185 .

Spaans, K., Hooper, A., 2016. InSAR processing for volcano monitoring and other near-real time applications. Journal of Geophysical Research: Solid Earth, 121(4), 2947-2960.

Tang, W., Motagh, M., Zhan, W., 2020. Monitoring active openpit mine stability in the Rhenish coalfields of Germany using a coherence-based SBAS method. International Journal of Applied Earth Observation and Geoinformation, 93, 102217.

Weiss, J. R., Walters, R. J., Morishita, Y., Wright, T. J., Lazecky, M., Wang, H., Hussain, E., Hooper, A. J., Elliott, J. R., Rollins, C. et al., 2020. High-resolution surface velocities and strain for Anatolia from Sentinel-1 InSAR and GNSS data. Geophysical Research Letters, 47(17), e2020GL087376.

Yunjun, Z., Fattahi, H., Amelung, F., 2019. Small baseline InSAR time series analysis: Unwrapping error correction and noise reduction. Computers \& Geosciences, 133, 104331. 